\title{
Photorefractive Ultrasonic Sensor for Weld Quality Monitoring
}

\author{
S. Zamiri*, M. Salfinger, M. Gruber, M. Stückler, B. Reitinger \\ Research Center for Non-Destructive Testing GmbH (RECENDT), Linz, Austria \\ Email: *saeid.zamiri@recendt.at
}

How to cite this paper: Zamiri, S., Salfinger, M., Gruber, M., Stückler, M. and Reitinger, B. (2017) Photorefractive Ultrasonic Sensor for Weld Quality Monitoring. Open Access Library Journal, 4: e3597. https://doi.org/10.4236/oalib.1103597

Received: April 12, 2017

Accepted: May 23, 2017

Published: May 26, 2017

Copyright $\odot 2017$ by authors and Open Access Library Inc.

This work is licensed under the Creative Commons Attribution International License (CC BY 4.0).

http://creativecommons.org/licenses/by/4.0/

\begin{abstract}
We report on a laser ultrasonic receiver based on two wave mixings in two nonlinear $\mathrm{Bi}_{12} \mathrm{SiO}_{20}(\mathrm{BSO})$ photorefractive crystals and its application for welded joints inspection. The nonlinear photo refractive crystals are operated with inverted voltages in respect to each other. Reversal of the voltage inverts the polarity of detected ultrasonic pulses which are generated by a pulsed laser on a sample surface. Present noise is, however, not inverted. Subtracting both signals after the two photorefractive crystals using a balanced photo-detector leads to suppression of noise and to an increase in the signal/noise ratio. We demonstrate the working principle by using a phase modulator and present laser ultrasonic measurement and compare the results from the proposed technique with a conventional two wave mixing interferometer based on single BSO crystal showing that the signal to noise/rate is significantly enhanced. Finally, we show the experimental results of application of these detectors for defects detection in two industrial samples with welded area.
\end{abstract}

\section{Subject Areas \\ Composite Material, Material Experiment}

\section{Keywords}

Photorefractive Crystal, Laser Ultrasonic, Weld Joints, Defect

\section{Introduction}

For contactless detection of ultrasonic waves several interferometers such as confocal Fabry-Perot, Michelson, and long path difference [1] interferometers have been applied. Each of them has its individual advantages and disadvantages concerning, e.g., frequency responses and sensitivity. However, most of these interferometers work best on mirror-like surfaces and exhibit reduced sensitivity on rough surfaces (e.g. welded joints). Also these kinds of interferometer are 
sensible to external noise as air fluctuation, sample vibrations or thermal deformations, thus requiring relatively complex stabilization techniques. This hinders their applicability in industrial applications such as crack detection and welded joints inspection with harsh environmental conditions. As an alternative to the before mentioned techniques interferometer based on nonlinear photorefractive crystals (PRC) have been established. A typical two wave mixing interferometer (TWMI) configuration enables broadband ultrasonic measurements on rough surfaces [2].

In a PRC ultrasound receiver, a reference beam with a planar wave front and an objective beam reflected from a sample surface having a distorted wave front interfere in a PRC [2] [3] [4]. Because of the nonlinear photorefractive effect the refractive index of the crystal gets spatially modulated and a dynamic hologram is formed. As a result a fraction of each beam is diffracted into direction of the other beam with its wave front matched to the transmitted beam. Especially, the planar reference beam is diffraction into direction of the speckled signal beam and both beams exhibit the same speckled wave front. Both beams are brought to interference on a fast photodiode. Thereby high frequency changes in the signal beam are demodulated if the changes are faster than the adaptability of the PRC. On the other hand, signals which are slower than the adaptability are suppressed caused by the dynamic formation of the hologram.

Typically, a PRC adapts on a time scale of a few milliseconds or even microseconds [2] [3] [4] resulting in effective suppression of low frequency noise from external sources. However, high frequency noise originating from a pulsed generation laser or from the detection laser is not suppressed. In this work we report on a low noise balanced laser ultrasonic (BLUS) system which can reject the common external noise. The setup is based on balanced detection using two PRCs [5]. Ina quasi-balanced interferometer using a single BSO crystal was demonstrated by utilizing microcontrollers to invert the high voltage on the crystal after each measurement [6]. Thus always two individual measurements were necessary for operation.

In the proposed BLUS receiver for welded joints inspection, two BSO crystals are utilized in drift regime but with reversed electric field in respect each other. By reversing the external electric field, the polarity of the ultrasonic pulses is inversed but the polarity of external noise is unaffected. By using a balanced photo-receiver the two outputs from both BSO crystals are subtracted and external noise is rejected. Thus the setup not only enables noise suppression from a single measurement but also reduction of noise caused by amplitude and phase fluctuations of the detection laser.

\section{Experiments and Discussion}

In Figure 1(a), the experimental setup for demonstrating the detection principle is shown. For detection of ultrasonic signals a CW laser with a wavelength of 532 $\mathrm{nm}$ (Klastech Symphony, $300 \mathrm{~mW}$ ) was used. The laser beam passed through a half wave plate HWP and a polarizing beam splitter PBS. By changing the 


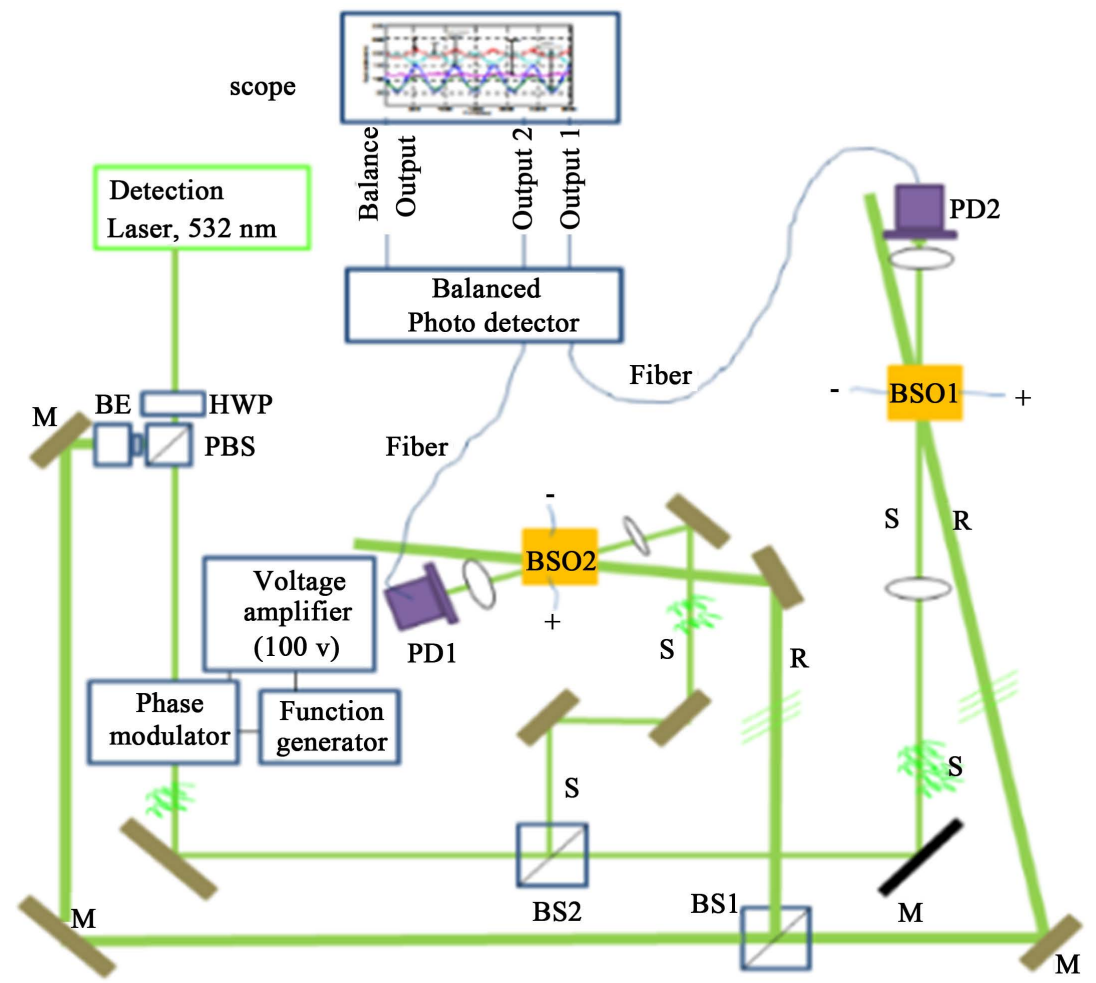

(a)

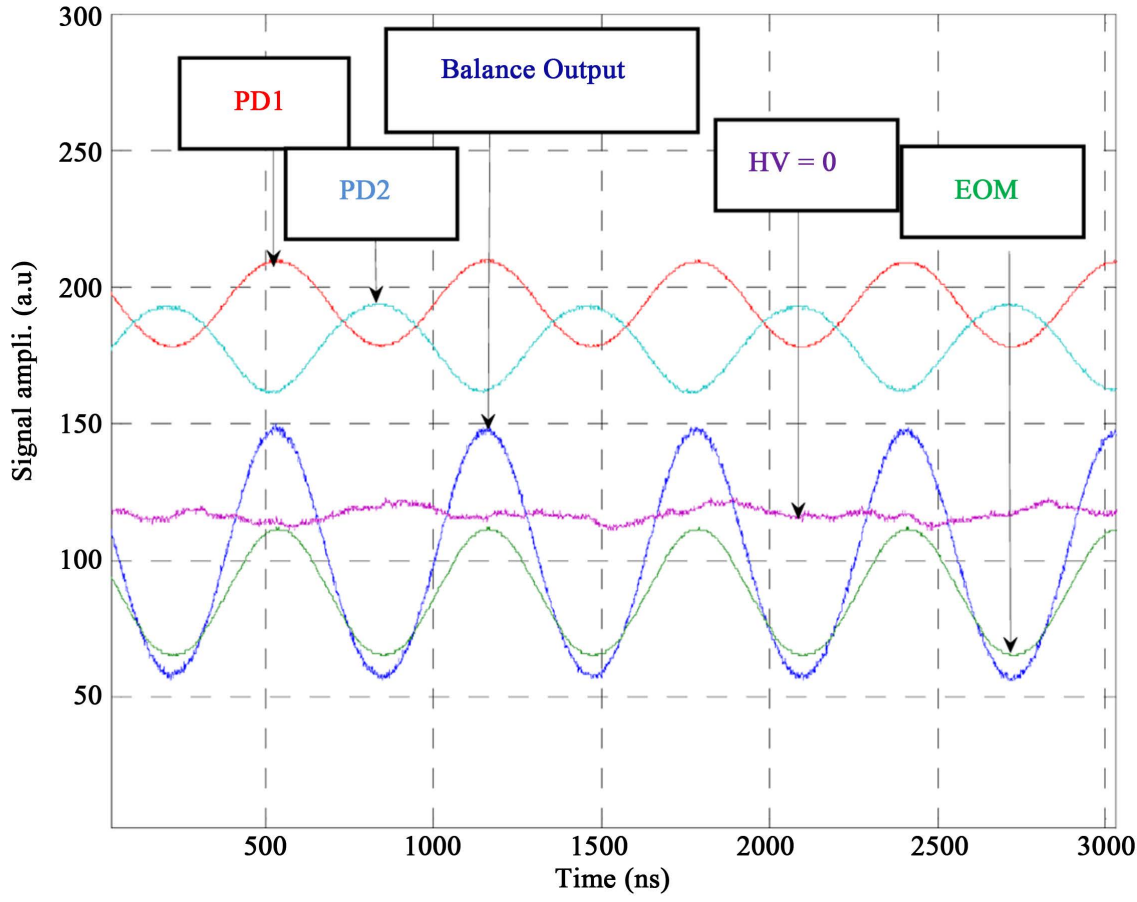

(b)

Figure 1. (a) Schematic of the balanced receiver for demonstrating the detection principle: EOM phase modulator, HWPs : half wave plate for $532 \mathrm{~nm}$ and $1064 \mathrm{~nm}$ wavelength, QWP: quarter wave plate, PBS: polarizer beam splitter, M: mirror, L: lens, S and R: signal and reference beam, BSO: photorefractive crystal, PD1 and 2: collimators, BE: beam expander; (b) Detected signals with positive (on PD1), reversed (PD2) and without applied high voltage to the BSO crystals. 
alignment of HWP the intensity ratio of reference to signal beam could be set. The reference beam was expanded using a beam expander BE to cover the crystals from electrode to electrode. The expanded beam was directed by mirrors $\mathrm{M}$ and beam splitter $\mathrm{BS} 1$ to the two $\mathrm{Bi}_{12} \mathrm{SiO}_{20}$ crystals, both with dimensions of 5 $\mathrm{mm} \times 5 \mathrm{~mm} \times 5 \mathrm{~mm}$ and [110] crystallographic axes. In the signal beam path an electro-optic modulator (DC-5 MHz) in combination with a voltage amplifier (0 - $100 \mathrm{~V}$ ) and a function generator was used to modulate the phase of the signal beam, thus simulating an ultrasound wave. The phase modulated signal beam and the reference beam were interfered in the crystals in which dynamic hologram gratings with lengths of a few micrometers were formed [7]. As a result fractions of the reference beams were diffracted in direction of the signal beams. The angle between signal and reference beams was about $10^{\circ}$.

To increase the local electric field $\left(E=E_{\text {diffusion }}+E_{\text {external }}\right)$ which increases the strength of the index grating $(\Delta n)$ and the diffraction efficiency $(\eta)$ [7] [8], an external DC high voltage was applied to each crystal. The voltage was applied in [001] direction by evaporated gold contacts with reversed polarity on the two crystals. The direction of the drift current depends on the direction of the applied external field. In a previous work [6] we presented that this leads to a relative shift between the two resulting space charge fields of $\pi$ if the external voltage is larger than the diffusion field but smaller than the saturation field of the crystal. This effect finally results in a phase shift between reference and refracted beam of $\pi / 2$ or $3 \pi / 2$ for positive or negative voltages, respectively. Therefore, the polarity of the detected ultrasound signal can be inverted by reversion of the external field [6].

In this work, $\mathrm{E}_{\text {external }}$ was set to $\pm 10 \mathrm{kV} / \mathrm{cm}$ in all experiments. After each crystal the respective collinear reference beam and signal beam were coupled into graded index fibers by using two collimators $(\mathrm{NA}=0.26, \mathrm{f}=34 \mathrm{~mm})$. The multimode fibers had a length of $1 \mathrm{~m}$ and were coupled to a broadband balanced amplified photo-detector (Thorlabs model PDB430A). By subtraction of the two ultrasonic signals the noise is reduced and thereby, the signal to noise ratio is increased.

In Figure 1(b) signals generated by the EOM are displayed for positive (PD1), reversed (PD2) and without applied high voltage on the BSO crystals. As expected, the detected ultrasound signal has very low amplitude in diffusion regime $(\mathrm{HV}=0)$ and applying high voltage to the BSO crystals increases the amplitude of the detected ultrasonic signals. From Figure 1(b) it can be seen that the polarity of the signal is reversed for the two different directions of electric field. The amplitude of the balanced detector is about two times is higher than the amplitudes of the individual detectors and has the same phase as the driving signal. In this experiment the applied voltage in the EOM was $50 \mathrm{~V}$. The response time of a PR interferometer is given by $\tau=1 /\left(2 \pi \mathrm{f}_{\text {cut }}\right)$ where $f_{\text {cut }}$ is the cut off frequency where the response drops to half of the maximum saturation value [9]. In our experiments the response times $(\tau)$ of the BLUS setup was measured to be about $1 \mathrm{~ms}$ to $3 \mathrm{~ms}$ depending to the beam intensity on the BSO crystals. 
To investigate BLUS in a realistic application for welded joints inspection, we performed LUS experiments as schematically depicted in Figure 2(a). Ultrasonic waves were generated by using a pulsed laser focused onto the surface of a metallic work piece. As sample a $3 \mathrm{~mm}$ thick aluminum plate was used. The sample

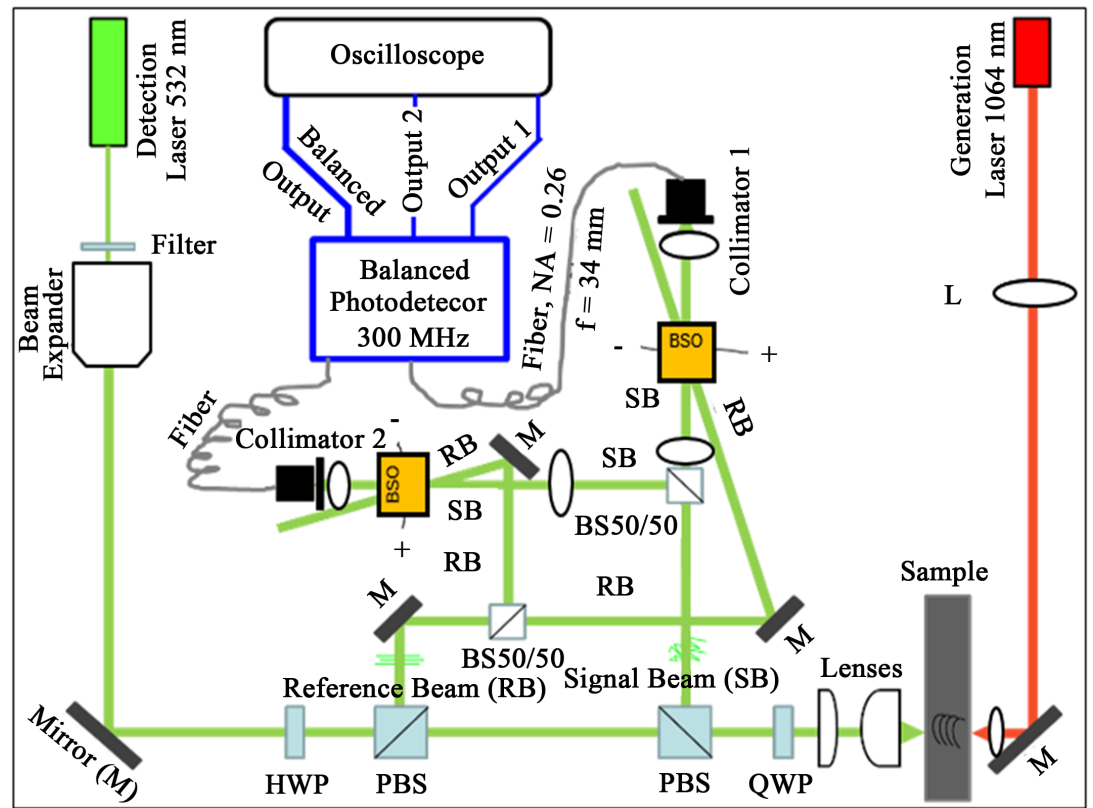

(a)

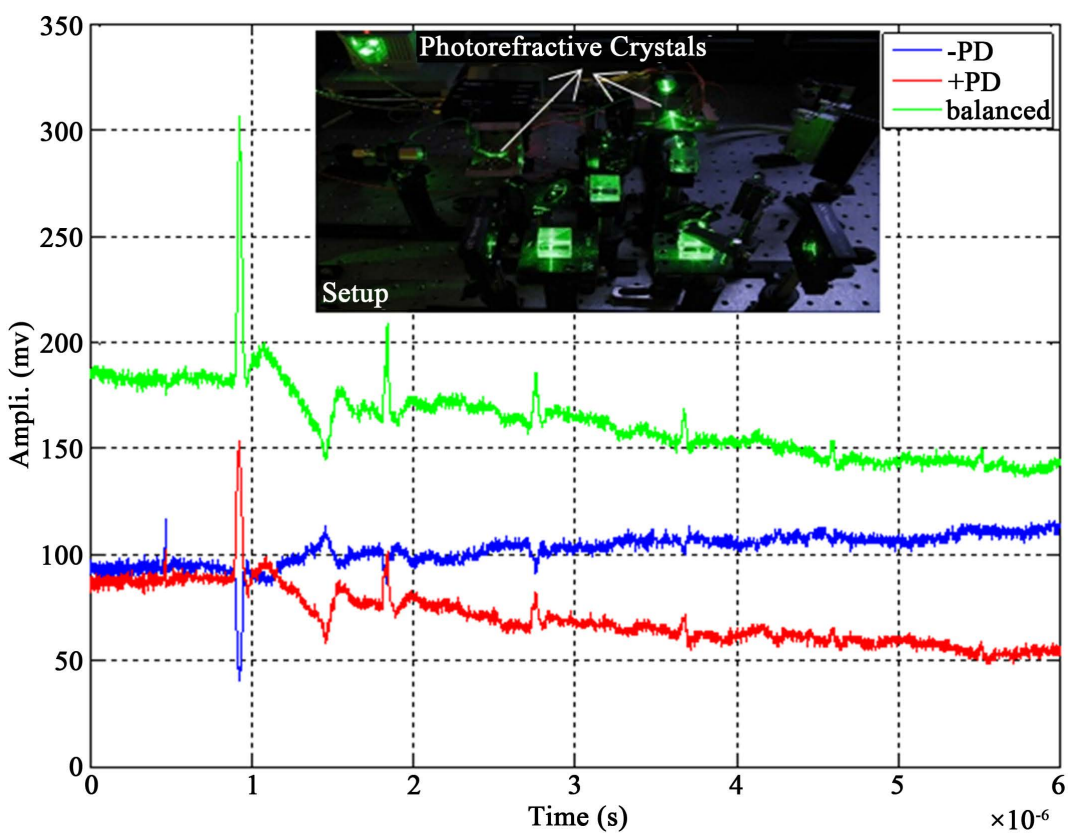

(b)

Figure 2. (a) Schematic of the balanced LUS receiver. HWP: half wave plate for $532 \mathrm{~nm}$ wavelength, QWP: quarter wave plate, PBS: polarizer beam splitter, M: mirror, L: lens, SB and RB: signal and reference beam, BSO: photorefractive crystal; (b) Measured ultrasonic signals(A-scan) detected in transmission geometry on an aluminum sheet with $3 \mathrm{~mm}$ thickness on output $1\left(-\mathrm{PD}\right.$, positive $\left.\mathrm{E}_{0}\right)$, output $2\left(+\mathrm{PD}\right.$, reversed $\left.\mathrm{E}_{0}\right)$ and with the balanced output, (Inset photo) Photograph of the BLUS interferometer. 
exhibited a rough surface to demonstrate the applicability of BLUS for nondestructive testing applications. For ultrasonic wave generation a Q-switched Nd: YAG pulsed laser with a maximum energy of $80 \mathrm{~mJ}$ and 20 ps pulse length at a wavelength of $1064 \mathrm{nmwas}$ utilized. Picoseconds pulses with $10 \mathrm{~mJ}$ energy were focused on the backside of the sample by means of a lens ( $f=100 \mathrm{~mm}$ ) and the ultrasonic signals were detected on the opposite side of the sample.

For detection of the ultrasonic pulses a CW Nd: YAG laser (Cobolt Symphony) with a power of $1000 \mathrm{~mW}$ and a wavelength of $532 \mathrm{~nm}$ was used. The reference beam RB was divided in two parts of equal power by using a beam splitter (BS50/50). The two beams were directed to the two crystals, BSO1 and BSO2, by means of mirrors. The signal beam SB was focused on the sample surface using a pair of lenses after passing a quarter wave plate QWP. The reflected signal beam was collected by the same lens systems and was deflected by the polarizing beam splitter PBS2 after passing QWP a second time. The signal beam was then divided in two equal parts by a beam splitter (BS50/50). Both signal beams were focused in the respective BSO crystals, BSO1 and BSO2. Finally, the diffracted reference beams and the transmitted signal beams were focused on the balanced detector at input 1 and input 2. Figure 2(b) shows a typical ultrasonic signal containing the direct longitudinal and transversal waves as well as several echoes of the longitudinal wave obtained by 32-time averaging. As can be seen the polarity of detected ultrasound signals is reversed for the two BSO crystals. The signal at $500 \mathrm{~ns}$ is the noise originating from triggering of the picosecond generation laser and has therefore the same polarity in both signals. In the balanced receiver the signals from input1 (BSO1) input2 (BSO2) are subtracted and on the output of the receiver the disturbance is almost suppressed. The output signal also exhibits higher amplitudes than the two individual channels at a lower noise level. From the time of flight of the echoes( 930 ns) and the know longitudinal sound velocity in aluminum $(6400 \mathrm{~m} / \mathrm{s})$ a sample thickness of $2.99 \mathrm{~mm}$ was calculated, which fits to the nominal $3 \mathrm{~mm}$ thick sample.

To compare BLUS with a conventional TWM setup (based on single crystal) [6], we measured the noise amplitude of both setups for different detection power under similar experiment conditions. A half wave plate and a polarizing beam splitter were used to change the laser power. In Figure 3(a) time traces obtained with the balanced setup are shown and the noise amplitude for both setups under the similar experimental conditions as a function of collected laser power was measured by using a spectrum analyzer. As is seen in Figure 3(b), the noise amplitude is proportional to the collected beam power at the balanced photo-detector and the noise amplitude in the balanced setup is lower than that of in conventional setup.

\section{Realistic Industrial Application: Quality Inspection of Welded Joints}

Laser ultrasound technology can be used to detect discontinues points in a welded area, an example of an important industrial application. To investigate 


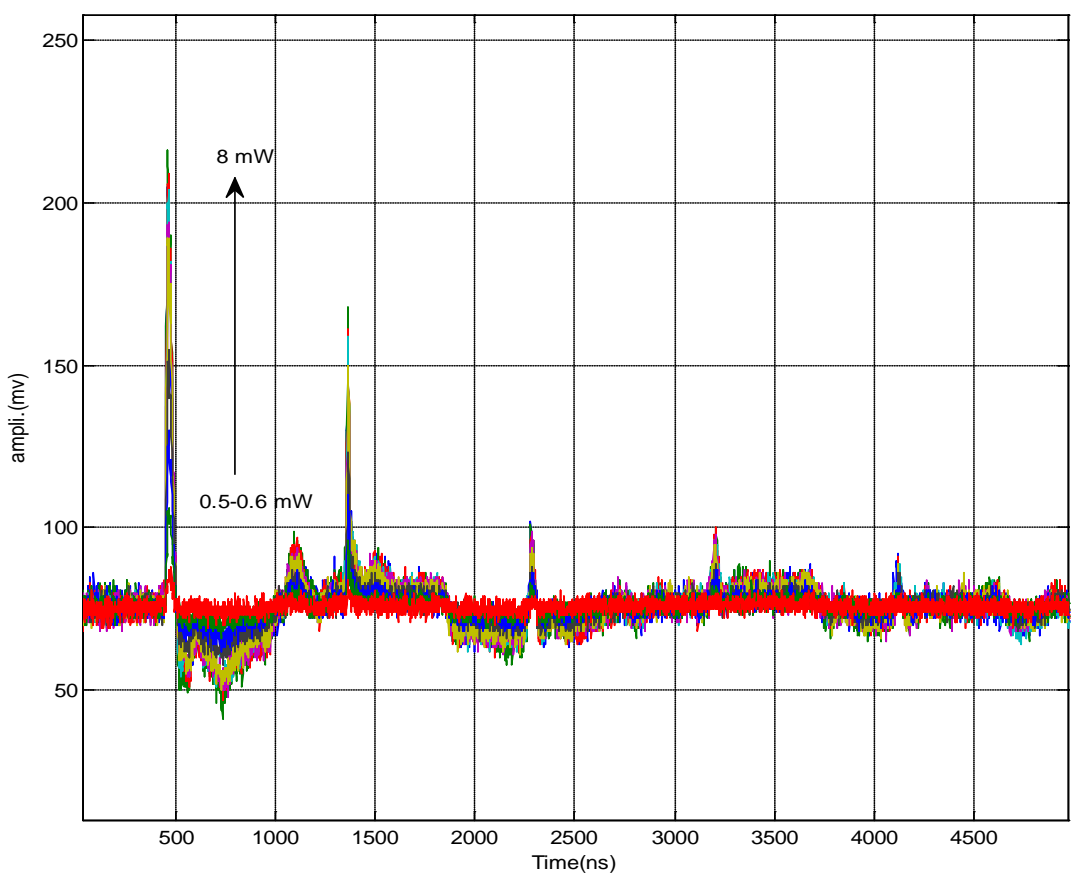

(a)

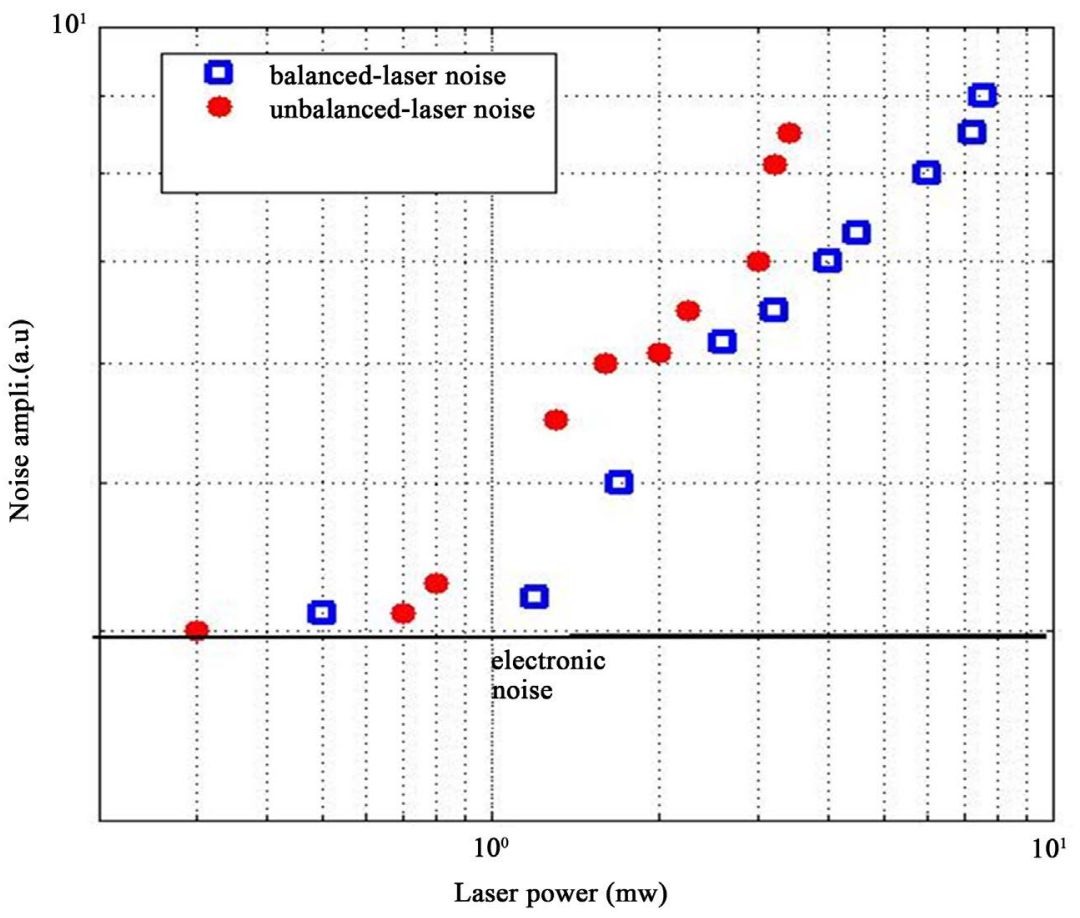

(b)

Figure 3. (a) Ultrasonic signals detected by the balanced setup as a function of collected laser power; (b) Noise voltage (Vrms) for a balanced and an unbalanced TWM setup under the similar experimental conditions as a function of collected laser power on the photo-detector.

the potential of LUS, the balanced setup was used to detect and characterize welded area in two different industrial specimens with dissimilar forms. The first industrial sample as shown in Figure 4(a) was used to investigate the potential 


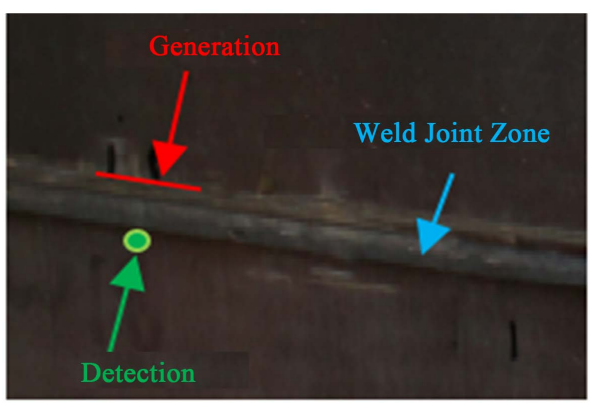

(a)

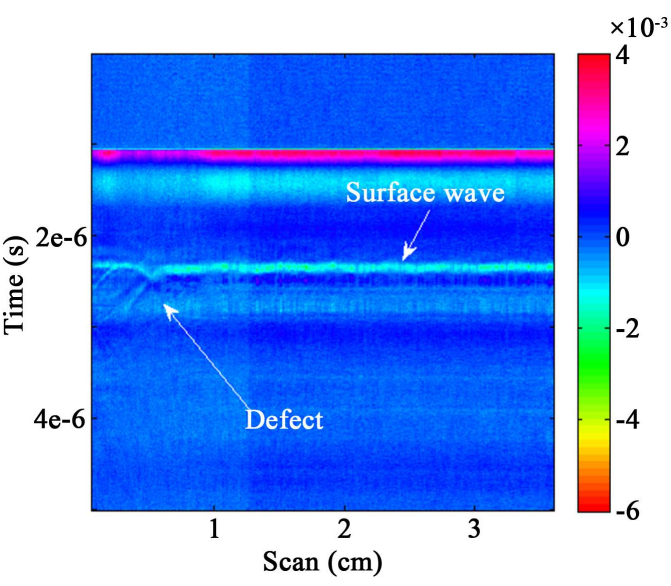

(b)

Figure 4. (a) Photograph of first industrial sample with welded joints, two steel plates with $4 \mathrm{~mm}$ thickness were welded together in a length of about $20 \mathrm{~cm}$; (b) B-scan and defects detection in the welding joints between two steel plates by using BLUS interferometer.

of the setup. Two steel plates with $4 \mathrm{~mm}$ thickness were welded together in a length of about $20 \mathrm{~cm}$. An electronic stage was used to scan the welded area between two plates to detect the disconnections or non-welded zone. The balanced set up was used for ultrasound pulse detection and the detection point was focused on the down side of the weld joint line. For the generation of the surface waves, picosecond pulses were focused in a $1 \mathrm{~cm}$ line by using a cylindrical lens with $150 \mathrm{~mm}$ focal length on the up side of the weld line as shown in Figure 4(b). The generation energy was set about $50 \mathrm{~mJ}$ at $10 \mathrm{~Hz}$.

As shown in the B-scan map of the scanned area of the first sample in Figure 4(b), the surface waves detected by the balanced setup in the disconnected zones are seen as scattered pulses with a parabolic shape. These clear scattering shown by vectors, display the position of the defects between two welded plates. The second sample was a cylinder pressure sensor integrated in the glow plug with welded joints which is seen in Figure 5(a) with generation-detection points with spot size of about $100 \mu \mathrm{m}$ and welded area in between. For this sample because of the curved surface, alignment of BLUS detector with similar reference and signal power on both PR crystals is difficult and therefore, we used the conventional LUS detector based on single BSO crystal for inspection of the welded area. By using an automatic LabVIEW program and synchronizing between the 


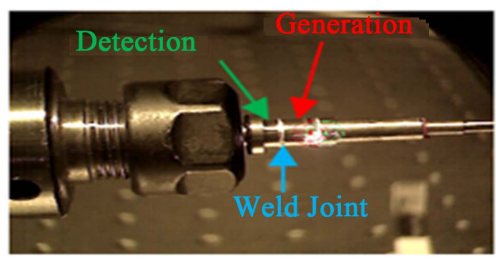

(a)

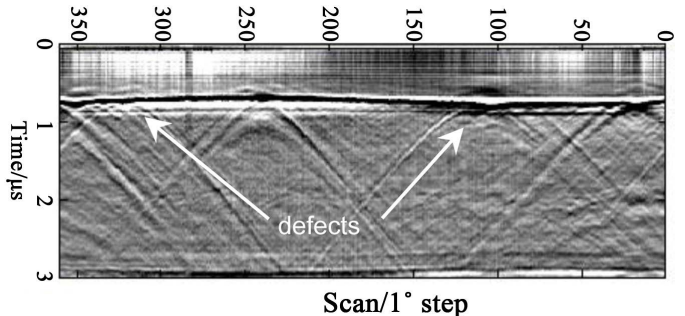

(b)

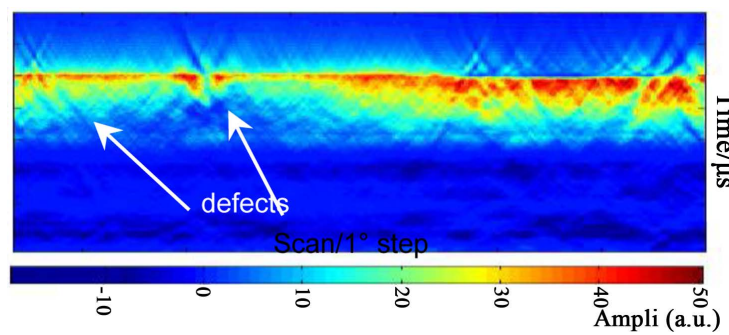

(c)

Figure 5. (a) Photograph of second industrial sample which is an innovative cylinder pressure sensing technology integrated in the glow plug with welded area; (b) Detecting defects in the welding joints by using simple TWM interferometer; (c) Reconstruction of defects.

generation laser, rotating the stepping motor, and Lecroy oscilloscope, we were able to rotate the sample $360^{\circ}$ in step of $1^{\circ}$ or even smaller $\left(0.5^{\circ}\right)$. For every point (A-scan), we detected the ultrasonic waves. Finally as is seen in Figure 5(b), we plot a B -scan which in the $\mathrm{x}$-axis is the whole number of measurements around the sample for 360 points and the $y$-axis is the time of flight of the ultrasonic wave. As is shown, the clear surface wave close to $1 \mu$ s in the $y$-axis is obvious and the scattered waves in shape of parabolic reflection in four points from the inside defects are clear. Figure 5(c) shows the Synthetic Aperture Focusing Technique (SAFT) of reconstruction [10] for profiling the defects in one of these samples which in one can see the position of the defects.

\section{Conclusion}

We demonstrated a new LUS receiver based on TWM in two BSO crystals. Both crystals are operated in drift regime with inverted external fields and reversing the drift direction changes the polarity of the LUS echoes but does no affects external noise. In this work, the detection principle by using an EOM and real measurements on an aluminum sample was presented. Furthermore, we examined the noise amplitudes for the balanced receiver and for a conventional unbalanced TWM as a function of collected detection laser power on the photo- 
detector, showing that the balanced receiver has a lower noise level. The proposed LUS receivers, BLUS and conventional detector for a realistic industrial application on rough surfaces for welded joints quality inspection were used and results showed the significant potential of these TWM sensors for non-destructive defects detection.

\section{Acknowledgements}

This work has been supported by European Project of COMBILASER, COMBI nation of non-contact, high speed monitoring and non-destructive techniques applicable to LASER Based Manufacturing through a self-learning system, with Grant No. 636902.

\section{References}

[1] Scruby, C.B. and Drain, L.E. (1990) Laser Ultrasonics: Techniques and Applications. Adam Hilger, Bristol.

[2] Honda, T., Yamashita, T. and Matsumoto, H. (1995) Optical Measurement of Ultrasonic Nanometer Motion of Rough Surface by Two-Wave Mixing in $\mathrm{Bi}_{12} \mathrm{SiO}_{20}$. Japanese Journal of Applied Physics, 34, 3737. https://doi.org/10.1143/JJAP.34.3737

[3] Blouin, A. and Monchalin, J.P. (1994) Detection of Ultrasonic Motion of a Scattering Surface by Two-Wave Mixing in a Photorefractive GaAs Crystal. Applied Physics Letters, 65, 932. https://doi.org/10.1063/1.112153

[4] Pouet, B.F., Ing, R.K., Krishnaswamy, S. and Royer, D. (1996) Heterodyne Interferometer with Two-Wave Mixing in Photorefractive Crystals for Ultrasound Detection on Rough Surfaces. Applied Physics Letters, 69, 3782. https://doi.org/10.1063/1.116997

[5] Reitinger, B., Zamiri, S., et al. (2014) Method and Device for Laser-Optical Detection of a Surface Movement of a Sample. Patent, WO2014066922A1.

[6] Hochreiner, A., Reitinger, B., Bouchal, K.D., Zamiri, S., Burgholzer, P. and Berer, T. (2013) Quasi-Balanced Two-Wave Mixing Interferometer for Remote Ultrasound Detection. Journal of Modern Optics, 60, 1327-1331. https://doi.org/10.1080/09500340.2013.837981

[7] Günter, P. and Huignard, J.P. (2007) Photorefractive Material and Their Applications. Springer Series, Vol. 115.

[8] Saleh, B.E.A. and Teich, M.C. (2007) Fundamental of Photonics. John Wiley \& Sons, USA.

[9] Girolamo, S.D. and Kamshilin, A.A. (2007) Fast Adaptive Interferometer on Dynamic Reflection Hologram in CdTe:V. Optics Express, 15, 545-555. https://doi.org/10.1364/OE.15.000545

[10] Blouin, A., Levesque, D., Neron, C., Enguehard, F., Drolet, D. and Monchalin, J.-P. (1998) Review of Progress in Qualitative Nondestructive Evaluation. Vol. 17, Plenum Press, New York. 
Submit or recommend next manuscript to OALib Journal and we will provide best service for you:

- Publication frequency: Monthly

- 9 subject areas of science, technology and medicine

- Fair and rigorous peer-review system

- Fast publication process

- Article promotion in various social networking sites (LinkedIn, Facebook, Twitter, etc.)

- Maximum dissemination of your research work

Submit Your Paper Online: Click Here to Submit

Or Contact service@oalib.com 